\title{
CALIDAD DE VIDA EN PACIENTES CON TUMORES CEREBRALES
}

\author{
Autora: Ana Sanz Cortés \\ Tipo de trabajo: Tesis Doctoral \\ Directores: Dra. María Eugenia Olivares Crespo y Dr. Juan Antonio Barcia Al- \\ bacar \\ Centro: Facultad de Psicoogía. Universidad Compluense de Madrid \\ Fecha de lectura: 18 de diciembre de 2016 \\ E-mail: asanz@imoncology.com
}

El presente trabajo se incluye dentro del limitado número de investigaciones que analiza la calidad de vida y las variables psicológicas más importantes de los pacientes con glioma de forma previa a su exéresis quirúrgica. El objetivo general ha sido determinar el efecto del tumor en los aspectos psicológicos más relevantes de estos pacientes, sin la influencia de otros factores como pueden ser los tratamientos oncológicos o los fármacos de soporte. Además se ha pretendido conocer cómo evolucionan estas variables psicológicas una vez que la situación clínica de los pacientes cambia por la aplicación de exéresis quirúrgica y la adición de los tratamientos oncológicos adyuvantes.

Por ello, se diseñó un estudio longitudinal con tres momentos de medida, el primero antes de la cirugía que permite obtener el efecto del tumor en la calidad de vida y las diferentes variables psicológicas valoradas, sin que otros factores clínicos y de los tratamientos puedan influir. El segundo momento de medida a los tres meses de la cirugía, coincide con el fin de la radioterapia en los pacientes que recibieron este tratamiento. $\mathrm{Y}$ el último momento de medida coincide con los seis meses de la cirugía para la resección del glioma.

La muestra inicial estaba compuesta por 41 participantes con sospecha de glioma del Servicio de Neurocirugía del Hospital Clínico San Carlos. Antes de la cirugía, los problemas más concretos de la calidad la calidad de vida de esta población fueron el malestar asociado a la menor participación familiar (56,1\%), dificultad en la expresión de pensamientos $(48 \%)$ y la frustración por un menor rendimiento (44\%). La calidad de vida global y la relativa a las dimensiones familiar-social y funcional son menores en los pacientes con glioma antes de la cirugía, frente a las poblaciones referenciales.

Respecto a los síntomas de ansiedad se hallan cifras son menores $(7,3 \%)$ a los referidos en la literatura, mientras que los valores para los síntomas de depresión son similares (14,6\%).

Los datos del rendimiento neurocognitivo ponen de manifiesto que antes de la cirugía todos los pacientes presentan una ejecución por debajo de los valores normativos para todos los dominios valorados. Cuando se analiza la presencia de déficits clínicos al menos el $40 \%$ de la muestra presenta 
problemas clínicos para la mayor parte de procesos. La variabilidad de los resultados pone de manifiesto que el tumor no afecta por igual a las diferentes funciones neurocognitivas, siendo el dominio más deterioro la atención alternante $(87,8 \%)$, y el que menos la generación de alternativas (22\%).

Otro de los objetivos fue analizar la relación entre la calidad de vida, ansiedad y depresión y el rendimiento neurocognitivo. Antes de la cirugía, se observa una fuerte relación entre los síntomas de depresión y la calidad de vida tanto global como en cada dimensión; así como con algunas de las principales funciones neurocognitivas (atención, lenguaje, memoria y funciones ejecutivas). Para la calidad de vida se evidencia que una mayor valoración global se asocia con un mejor rendimiento en cada uno de los procesos de cada dominio neurocognitivo. Además, se halla una relación positiva entre la valoración subjetiva de los problemas cognitivos y manifestaciones conductuales con su rendimiento en pruebas neuropsicológicas. De todo esto, podría inferirse que estos pacientes no sobreestiman de sus dificultades.

De igual forma se analizó la influencia de algunos de los factores moduladores, recogidos en la literatura, en las principales variables a estudio:

- Calidad de vida: el género explica diferencias en la calidad de vida funcional, de tal forma que las mujeres se encuentran más activas en las áreas de su vida diaria y están más satisfechas. En contra de lo esperado, las crisis epilépticas se asocian con una mejor calidad de vida global y en la dimensión familiarsocial.
- Ansiedad y depresión: el grado de malignidad del glioma explica diferencias en los síntomas de ansiedad, siendo los pacientes con gliomas de bajo grado quienes presentan un mayor número de síntomas.

- Rendimiento neurocogntivo: queda demostrada la relación inversa entre el rendimiento en las pruebas neuropsicológicas y la edad, así como con el nivel educativo. Además, el grado de malignidad del glioma afecta desde el primer momento al rendimiento en funciones ejecutivas.

En los momentos posteriores a la cirugía, a los tres y seis meses, la muestra se redujo a 29 y 28 participantes respectivamente, aunque se mantienen las características sociodemográficas y médico-clínicas de la población. Los problemas más frecuentes de la calidad de vida continúan siendo el malestar y la frustración por no poder realizar las actividades funcionales, con cifras superiores al $50 \%$.

Después de la cirugía, la calidad de las dimensiones familiar-social y funcional y la valoración global de estos pacientes sigue siendo peor que en las poblaciones referenciales. De estos datos se deriva que el propio tumor genera dificultades que afectan directamente al funcionamiento habitual en esta población. Además, estos enfermos están menos satisfechos con el apoyo percibido, tanto por parte de la familia como del grupo de amigos. Y en último lugar, su calidad de vida global está determinada por las limitaciones en las dimensiones familiar-social y funcional, y no por las relativas al área emocional. 
A los tres meses de la cirugía se observa una menor calidad de vida en la dimensión física y específica de los tumores cerebrales frente a la medida previa, que se mantiene a los seis meses. Así, los efectos neurotóxicos de la radioterapia junto con los propios de la quimioterapia oral, pueden ser los responsables de que permanezca el deterioro a nivel físico y el aumento de las manifestaciones específicas de la localización tumoral.

Respecto a los casos clínicos para la ansiedad y la depresión, a los tres meses de la cirugía se observa una disminución de los porcentajes (5\% ansiedad y 9,8\% depresión); que a los seis meses alcanzan valores similares a los referidos para la población oncológica ( $10 \%$ ansiedad y $25 \%$ depresión). Cuando se analiza la evolución de los síntomas de ansiedad y depresión, los datos indican una disminución de los síntomas de ansiedad progresivamente en las dos medidas posteriores a la cirugía; mientras que para los síntomas de depresión se evidencia un mantenimiento durante los primeros seis meses de enfermedad.

A los tres y a los seis meses de la cirugía el rendimiento neurocognitivo sigue siendo menor frente a los valores normativos, excepto para memoria verbal y evocación categorial. Los datos de déficits clínicos mantienen la variabilidad en las cifras de acuerdo al dominio considerado. En ambas medidas la velocidad del procesamiento de la información y la atención alternante se definen como los procesos neurocognitivos más afectados. Por el contrario, disminuye el porcentaje de afectación en los procesos de memoria verbal, pudiendo explicarse por el efecto de la práctica al carecer el cuestionario de forma paralela.

En pacientes con gliomas de bajo grado no se observan cambios en el rendimiento de los dominios neurocognitivos entre la medida previa a la cirugía y los tres meses después de la misma. Y a los seis meses se contempla una mejoría en el funcionamiento del dominio del lenguaje respecto al momento previo a la cirugía, así como en la memoria verbal frente a las dos medidas anteriores. En pacientes con gliomas de alto grado a los tres meses se evidencia un empeoramiento en dominios atencionales y ejecutivos; mientras que al contrario se observa una mejoría en tareas de memoria visual inmediata. Para la medida de los seis meses, se aprecia que estos pacientes mantienen el peor rendimiento en funciones ejecutivas y la mejor ejecución en memoria visual inmediata.

Después de la cirugía se mantienen las relaciones entre las variables dependientes del estudio e incluso, adquieren una mayor fuerza a los seis meses. Los síntomas de depresión se asocian de forma negativa con todas las dimensiones de la calidad de vida y con todos los procesos neurocognitivos valorados; mientras que la calidad de vida global, la funcional y específica de tumores cerebrales se relacionan directamente con el rendimiento en todos los dominios valoraros.

Las variables moduladoras después de la cirugía influyen de forma diferente al momento previo:

- Calidad de vida: el grado de malignidad del glioma y el uso de corticoides se asocian de forma inversa con la calidad de vida a los seis meses de la cirugía. 
- Ansiedad y depresión: la historia psicopatológica previa influye en los síntomas de depresión a los tres meses, y la progresión de la enfermedad lo hace en los síntomas de ansiedad en la misma medida.

- Rendimiento neurocogntivo: el grado de malignidad influye en las funciones ejecutivas, habilidades viso-espaciales y memoria verbal a los tres meses; en tanto es determinante en el rendimiento de procesos atencionales y habilidades viso-espaciales a los seis meses. El uso de corticoides se asocia con un menor rendimiento en dominios atencionales y ejecutivos en ambos momentos posteriores a la cirugía. La progresión de la enfermedad influye en un menor rendimiento en procesos atencionales a los tres y seis meses de la cirugía. La lateralidad del glioma afecta únicamente a tareas verbales a los tres meses. El mapeo intraoperatorio durante la cirugía se asocia con un mejor rendimiento en tareas verbales y funciones ejecutivas a los seis meses.

Un último objetivo fue determinar el papel predictor de las variables psicológicas previas a la cirugía, en la calidad de vida a los seis meses. Se halló que los síntomas de depresión iniciales predicen tanto la valoración global como la relativa a las dimensiones funcional y específica de tumores cerebrales. El rendimiento en funciones ejecutivas antes de la cirugía determina la calidad de vida global a los seis meses; y las habilidades visoespaciales lo hacen para las dimensiones funcional y específica de tumores cerebrales.

Futuras líneas de investigación deberán soslayar los problemas metodológicos del presente estudio, referidos al limitado tamaño de la muestra, la ausencia de grupo control y variabilidad de los tratamientos oncológicos de la muestra. 